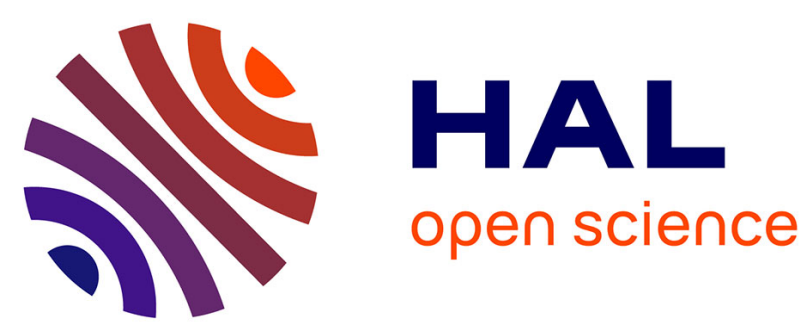

\title{
Non-linear behaviour of free-edge shallow spherical shells: Effect of the geometry
}

\author{
Cyril Touzé, Olivier Thomas
}

\section{To cite this version:}

Cyril Touzé, Olivier Thomas. Non-linear behaviour of free-edge shallow spherical shells: Effect of the geometry. International Journal of Non-Linear Mechanics, 2006, 41 (5), pp.678-692. 10.1016/j.ijnonlinmec.2005.12.004 . hal-00838885

HAL Id: hal-00838885

https://hal-ensta-paris.archives-ouvertes.fr/hal-00838885

Submitted on 18 Mar 2016

HAL is a multi-disciplinary open access archive for the deposit and dissemination of scientific research documents, whether they are published or not. The documents may come from teaching and research institutions in France or abroad, or from public or private research centers.
L'archive ouverte pluridisciplinaire $\mathbf{H A L}$, est destinée au dépôt et à la diffusion de documents scientifiques de niveau recherche, publiés ou non, émanant des établissements d'enseignement et de recherche français ou étrangers, des laboratoires publics ou privés. 


\title{
Non-linear behaviour of free-edge shallow spherical shells: Effect of the geometry
}

\author{
C. Touzéa ${ }^{\mathrm{a}, *}$, O. Thomas ${ }^{\mathrm{b}}$ \\ ${ }^{a}$ ENSTA-UME, Chemin de la Hunière, 91761 Palaiseau Cedex, France \\ ${ }^{\mathrm{b}}$ Structural Mechanics and Coupled Systems Laboratory, CNAM, 2 rue Conté, 75003 Paris, France
}

\begin{abstract}
Non-linear vibrations of free-edge shallow spherical shells are investigated, in order to predict the trend of non-linearity (hardening/softening behaviour) for each mode of the shell, as a function of its geometry. The analog for thin shallow shells of von Kármán's theory for large deflection of plates is used. The main difficulty in predicting the trend of non-linearity relies in the truncation used for the analysis of the partial differential equations (PDEs) of motion. Here, non-linear normal modes through real normal form theory are used. This formalism allows deriving the analytical expression of the coefficient governing the trend of non-linearity. The variation of this coefficient with respect to the geometry of the shell (radius of curvature $R$, thickness $h$ and outer diameter $2 a$ ) is then numerically computed, for axisymmetric as well as asymmetric modes. Plates (obtained as $R \rightarrow \infty$ ) are known to display a hardening behaviour, whereas shells generally behave in a softening way. The transition between these two types of non-linearity is clearly studied, and the specific role of 2:1 internal resonances in this process is clarified.
\end{abstract}

Keywords: Shallow spherical shells; Hardening/softening behaviour; Non-linear normal modes; Internal resonance

\section{Introduction}

Geometrically non-linear vibrations of shallow shells is a problem of widespread relevance, with a range of applications from aerospace industry to musical acoustics [1-3]. Despite numerous studies, some important features still remain partially or completely unsolved, due to the non-linear nature of the problem. One of the most common property of non-linear oscillations is the dependence of the frequency of free oscillation on vibration amplitude, which can be of the hardening or softening type. It is a well-known fact that flat plates display a hardening behaviour, as it has been shown both theoretically and experimentally (see e.g. [4-9]). Introducing an initial curvature in the middle surface of the structure creates a quadratic nonlinearity, which, in turn, may change the non-linear behaviour to softening type, depending on the balance of the magnitude of quadratic and cubic terms $[6,10,11]$. It is thus a legitimate question to determine the correct non-linear behaviour of shallow spherical shells, and more precisely, the transition from the hardening (flat plate) behaviour to the softening one, as the curvature increases.

Among the available studies concerned with this question, almost all of them restrict to the case of axisymmetric vibrations. Evensen and Evan-Iwanowski [12] found a softening behaviour with the harmonic balance method, without studying the transition from hardening to softening behaviour. Many investigators used a single-mode approach to study the effect of geometry on the non-linear behaviour: Grossman et al. [13] investigated different type of boundary conditions and mentioned the transition from hardening to softening behaviour as the riseto-thickness ratio increases, Yasuda and Kushida [14] found the first mode to be softening for a little curvature whereas the second stays of the hardening type. Singh et al. [15] and Sathyamoorthy [16] studied the influence of transverse shear deformation and rotatory inertia in the case of a moderately thick shell. Some slight improvements were proposed in order to overcome the single-mode truncation. Pandalai and Varadan 
[17] used a two-mode expansion, but for the precise transition from hardening to softening behaviour, restrict once again to a single-mode expansion [10]. Li [18] proposed a time-mode approach to solve the axisymmetrical vibrations, and studied the effect of orthotropy. Gonçalves [19] used three modes to derive free vibration results, but took only a few discrete values for the geometry, and didn't mention internal resonances. Other geometrical parameters have been studied, as the effect of geometrical imperfections on the type of non-linearity [20]. Leissa and Kadi [21] studied the transition for a shallow shell having a rectangular boundary. Doubly-curved shallow shells have also been investigated, by Shin [22] with the assumption of a single-mode, recently by Alhazza [23] with the direct method proposed by Nayfeh [24], and by Amabili [25] for a number of different geometries.

During the last decade, a number of different proofs showed that too severe truncations lead to erroneous results in the prediction of the trend of non-linearity. Nayfeh et al. [26] seem to be the first to point out the shortcomings of using a single-mode approach, which could lead to quantitative as well as qualitative erroneous results. The most direct solution is to keep a sufficient number of modes in the analysis, which renders analytical expressions almost intractable and leads to intensive numerical computations. This approach has been used by Amabili et al. [27-29] for the non-linear behaviour of circular cylindrical shells in order to clarify controversial results upon the trend of non-linearity (see e.g. [27,30-33]). They used a model with up to 23 degrees of freedom (dofs) [29], and highlight the fundamental role played by axisymmetrical contractions in asymmetrical vibrations. Moreover, Pellicano et al. [29] propose a map of non-linearity, showing the trend of non-linearity as a function of the two independent geometrical parameters of the shell, with a severely reduced models composed of three modes.

In order to avoid the main drawbacks associated to the large number of modes retained, significant efforts have been made toward definitions of reduced-order models (ROMs), able to predict the correct non-linear behaviour with a limited number of equations. A complete review of the available mathematical methods is provided by Steindl and Troger [34]. Strategies based upon the application of the multiple scales method directly into the PDE have been proposed by Nayfeh and coworkers [24,35], and has been successfully applied to the cases of non-linear vibrations of buckled beams [36,37], shallow suspended cables [38] and doubly-curved cross-ply shallow shells [23]. Non-linear normal modes (NNMs), defined as invariant manifolds in phase space [39], state a proper framework to embed the influence of all linear modes into a single NNM. It has been shown that the motion onto the invariant manifold, defined by a single oscillator equation, predicts the correct trend of non-linearity [40].

The objective of this paper is to derive the correct trend of non-linearity for axisymmetric as well as asymmetric nonlinear vibrations of shallow spherical shells, by using the framework of NNMs, defined through real normal form theory, as proposed in [40]. The paper is divided into three main parts: first, a non-linear model of the shell is briefly presented, relying on the analog for thin shallow shells of von Kármán's theory for large deflection of plates. A thorough presentation of the model as well as experimental validations are available in $[41,42]$. Then the framework of NNMs is presented and the analytical coefficient governing the trend of non-linearity is derived. Important comments from the analytical formula, with respect to modal truncation and 2:1 internal resonances, are given. Finally, results are presented for purely asymmetric modes, axisymmetric and mixed modes (asymmetric modes with at least one nodal circle).

\section{Governing equations}

The aim of this section is to provide the PDEs of motion for a shallow spherical shell. Only the main results are given, the interested reader is referred to [41] for more details on the non-linear model.

\subsection{Local equations}

A spherical shell of thickness $h$, radius of curvature $R$ and outer diameter $2 a$, made of a homogeneous isotropic material of density $\rho$, Poisson ratio $v$ and Young's modulus $E$, is considered (see Fig. 1). Large transverse deflections and moderate rotations are considered, so that the model is a generalization of von Kármán's theory for large deflection of plates. The main geometrical hypotheses, which are relevant for this study are the following:

- the shell is thin: $h / a \ll 1$ and $h / R \ll 1$;

- the shell is shallow: $a / R \ll 1$.

Other assumptions are classical for large deflection von Kármán's type models [41]. Finally, as this study is concerned with the trend of non-linearity, which is dictated by the conservative problem, damping and external forces are not taken into account. The equations of motion are given in terms of the transverse displacement $w$ along the normal to the mid-surface and the Airy stress function $F$, for all time $t$ :

$D \Delta \Delta w+\frac{1}{R} \Delta F+\rho h \ddot{w}=L(w, F)$,

$\Delta \Delta F-\frac{E h}{R} \Delta w=-\frac{E h}{2} L(w, w)$,

where $D=E h^{3} / 12\left(1-v^{2}\right)$ is the flexural rigidity, $\ddot{w}$ is the second partial derivative of $w$ with respect to time, $\Delta$ is the laplacian and $L$ is a bilinear quadratic operator. With the assumption of a shallow shell fulfilled, the spatial operators are written in polar coordinates, and thus reads:

$\Delta(\cdot)=(\cdot)_{, r r}+\frac{1}{r}(\cdot)_{, r}+\frac{1}{r^{2}}(\cdot)_{, \theta \theta}$,

and

$$
\begin{aligned}
L(w, F)= & w_{, r r}\left(\frac{F_{, r}}{r}+\frac{F_{, \theta \theta}}{r^{2}}\right)+F_{, r r}\left(\frac{w_{, r}}{r}+\frac{w_{, \theta \theta}}{r^{2}}\right) \\
& -2\left(\frac{w_{, r \theta}}{r}-\frac{w_{, \theta}}{r^{2}}\right)\left(\frac{F_{, r \theta}}{r}-\frac{F_{, \theta}}{r^{2}}\right) .
\end{aligned}
$$




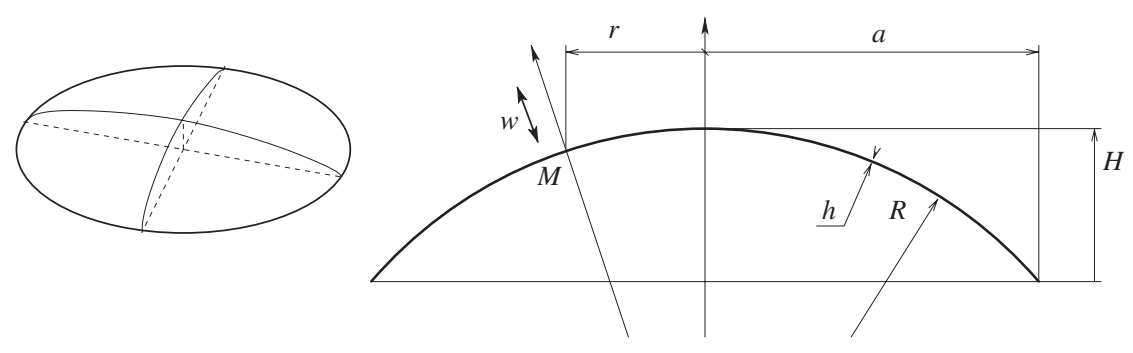

Fig. 1. Geometry of the shell: three-dimensional sketch and cross section.

Free-edge boundary conditions are considered:

$F$ and $w$ are bounded at $r=0$,

$F_{, r}+\frac{1}{a} F_{, \theta \theta}=0, \quad F_{, r \theta}+\frac{1}{a} F_{, \theta}=0$, at $r=a$,

$w_{, r r}+\frac{v}{a} w_{, r}+\frac{v}{a^{2}} w_{, \theta \theta}=0, \quad$ at $r=a$,

$w_{, r r r}+\frac{1}{a} w_{, r r}-\frac{1}{a^{2}} w_{, r}+\frac{2-v}{a^{2}} w_{, r \theta \theta}$

$$
-\frac{3-v}{a^{3}} w_{, \theta \theta}=0, \quad \text { at } r=a \text {. }
$$

The above equations stem from the vanishing of the external force at the edge: Eqs. (4b) are related to the membrane forces, Eq. (4c) to the bending moment and Eq. (4d) to the twisting moment and transverse shear force.

\subsection{Dimensionless form of the equations}

Dimensionless variables are introduced by

$r=a \bar{r}, \quad t=a^{2} \sqrt{\rho h / D} \bar{t}, \quad w=h \bar{w}, \quad F=E h^{3} \bar{F}$.

Substituting the above definitions in equations of motion, Eq. (1a,b), and dropping the overbars in the results, one obtains:

$\Delta \Delta w+\varepsilon_{\mathrm{q}} \Delta F+\ddot{w}=\varepsilon_{\mathrm{c}} L(w, F)$,

$\Delta \Delta F-\sqrt{\kappa} \Delta w=-\frac{1}{2} L(w, w)$

where the aspect ratio $\kappa$ of the shell has been introduced:

$\kappa=\frac{a^{4}}{R^{2} h^{2}}$

As it will be shown next, for a fixed value of the Poisson ratio $v$, all the linear results (eigenfrequencies and mode shapes), as well as the trend of non-linearity, only depend on $\kappa$, which is the only free parameter related to the geometry of the shell. The two other parameters $\varepsilon_{\mathrm{q}}$ and $\varepsilon_{\mathrm{c}}$ appearing in Eq. (6) are equal to

$\varepsilon_{\mathrm{q}}=12\left(1-v^{2}\right) \frac{a^{2}}{R h}=12\left(1-v^{2}\right) \sqrt{\kappa}, \quad \varepsilon_{\mathrm{c}}=12\left(1-v^{2}\right)$.

Their subscripts comes from the fact that they balance, respectively, the quadratic and the cubic terms in the non-linear ordinary differential equations governing the dynamics of the problem (see Eq. (11)).

\subsection{Linear analysis}

The linearized equations of motion are analyzed to derive the eigenmodes and eigenfrequencies of the problem, as a function of the geometry. The eigenmodes are the solutions of:

$\Delta \Delta \Phi+\chi \Delta \Psi-\omega^{2} \Phi=0$,

$\Delta \Delta \Psi=\Delta \Phi$,

where $\Phi$ refers to the eigenmodes of the transverse motion and $\Psi$ to those of the membrane motion. The coefficient $\chi=$ $12\left(1-v^{2}\right) \kappa$ is the only parameter of the linear problem. All the study could have been realized by taking $\chi$ as the geometrical parameter, as it is sometimes done by various authors [12,19]. However, the results will be presented as functions of $\kappa$, in order to set apart the material property which appear through the Poisson ratio $v$ in the expression of $\chi$. In the remainder of this study, $v$ is kept constant at $v=0.33$.

Transverse and membrane mode shapes are numbered $\Phi_{(k, n)}$ and $\Psi_{(k, n)}$ where $k$ is the number of nodal diameters and $n$ the number of nodal circles. Axisymmetric modes are such that $k=0$. For $k \geqslant 1$ (asymmetric modes), the associated eigenvalue has a multiplicity of two, so that for each eigenfrequency, there are two independent mode shapes, called preferential configurations or companion modes. Among these modes, purely asymmetric modes (such that $k \geqslant 2$ and $n=0$ ) are distinguished from mixed modes (such that $k \geqslant 1$ and $n \geqslant 1$ ).

The linear analysis provided in Ref. [41] shows that all deformed shapes, except membrane mode shapes for purely asymmetric modes, have a negligible dependence on the geometry. On the contrary, the eigenfrequencies dependence on the aspect ratio $\kappa$, represented on Fig. 2, shows different behaviour, which leads to classify the modes into two families.

The first family contains the purely asymmetric modes, since their eigenfrequencies display a slight dependence on curvature. The second family contains axisymmetric and mixed modes. They show a huge eigenfrequency dependence on curvature. Due to these different behaviours, the study of the trend of nonlinearity will distinguish: purely asymmetric modes, axisymmetric and mixed modes.

\subsection{Modal expansion}

The complete non-linear equations of motion (6) are projected onto the natural basis of the transverse eigenmodes. 


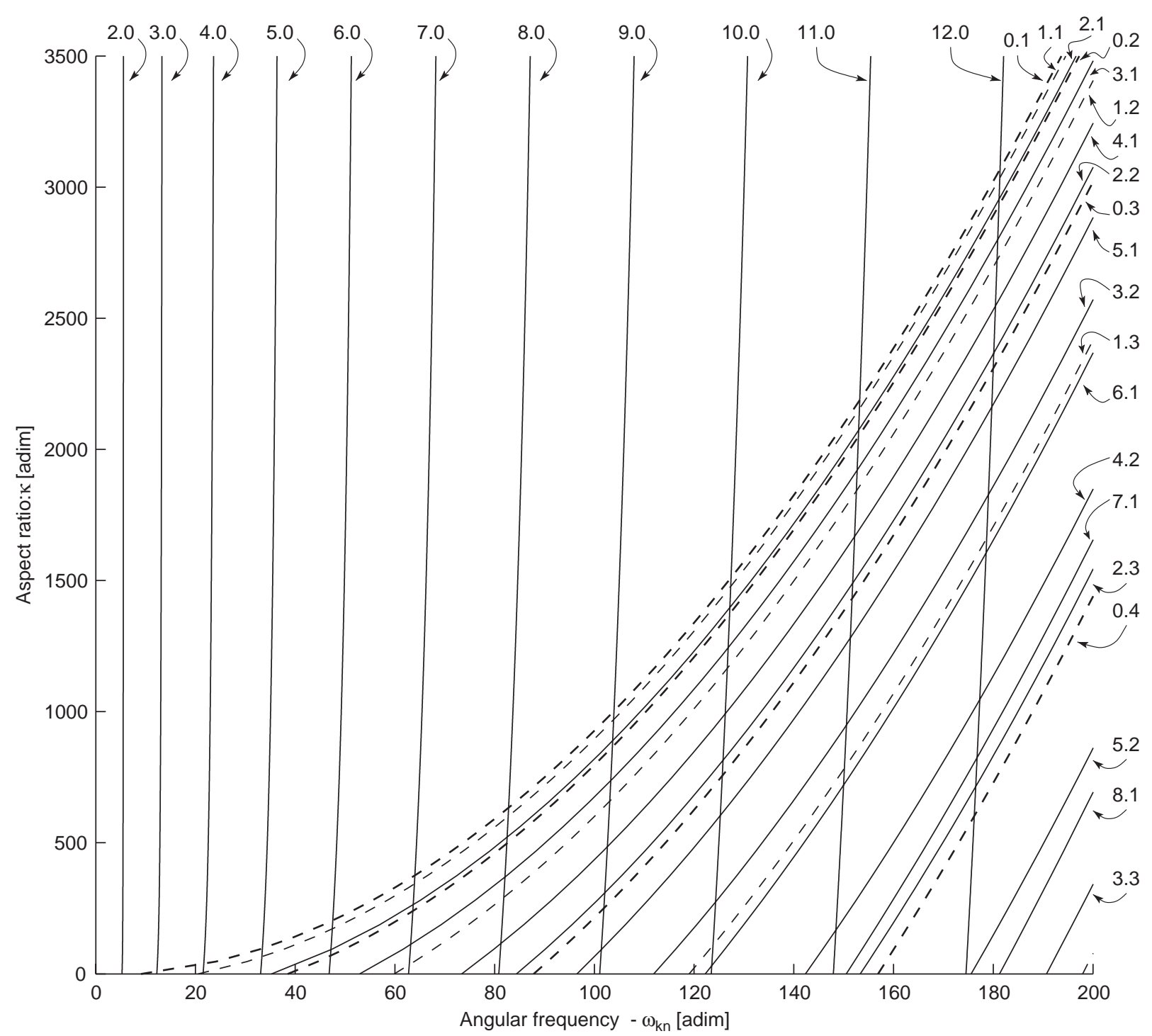

Fig. 2. Dimensionless natural frequencies $\omega_{k n}$ of the shell as a function of the aspect ratio $\kappa$, for $v=0.33$.

The displacement is thus written as:

$$
w(r, \theta, t)=\sum_{p=1}^{+\infty} X_{p}(t) \Phi_{p}(r, \theta),
$$

where the subscript $p$ refers to a specific mode of the shell, defined by a couple $(k, n)$ and, if $k \neq 0$, a binary variable which indicates the preferential configuration considered (sine or cosine companion mode). The modal displacements $X_{p}$ are the unknowns, and their dynamics is governed by $\forall p \geqslant 1$ :

$$
\begin{gathered}
\ddot{X}_{p}+\omega_{p}^{2} X_{p}+\varepsilon_{\mathrm{q}} \sum_{i=1}^{+\infty} \sum_{j=1}^{+\infty} \beta_{i j}^{p} X_{i} X_{j} \\
+\varepsilon_{\mathrm{c}} \sum_{i=1}^{+\infty} \sum_{j=1}^{+\infty} \sum_{k=1}^{+\infty} \Gamma_{i j k}^{p} X_{i} X_{j} X_{k}=0 .
\end{gathered}
$$

The expression of the non-linear coefficients are

$$
\begin{aligned}
\beta_{i j}^{p}= & -\iint_{\mathscr{S}_{\perp}} \Phi_{p} L\left(\Phi_{i}, \Psi_{j}\right) \mathrm{d} S-\frac{1}{2} \sum_{b=1}^{+\infty} \frac{1}{\xi_{b}^{4}} \\
& \times \iint_{\mathscr{S}_{\perp}} L\left(\Phi_{i}, \Phi_{j}\right) \Upsilon_{b} \mathrm{~d} S \iint_{\mathscr{S}_{\perp}} \Phi_{p} \Delta \Upsilon_{b} \mathrm{~d} S, \\
\Gamma_{i j k}^{p}= & \frac{1}{2} \sum_{b=1}^{+\infty} \frac{1}{\xi_{b}^{4}} \iint_{\mathscr{S}_{\perp}} L\left(\Phi_{i}, \Phi_{j}\right) \Upsilon_{b} \mathrm{~d} S \\
& \times \iint_{\mathscr{S}_{\perp}} \Phi_{p} L\left(\Phi_{k}, \Upsilon_{b}\right) \mathrm{d} S .
\end{aligned}
$$

The $\Upsilon_{n}$, as well as its associated zero $\xi_{n}$, are defined in [41]. $\mathscr{S}_{\perp}$ is the domain defined by $(r, \theta) \in\left[\begin{array}{ll}0 & 1\end{array}\right] \times\left[\begin{array}{ll}0 & 2 \pi\end{array}\right]$.

The temporal equations (11) describe the dynamics of the shell. In particular, the trend of non-linearity can be inferred from these equations. As shown by various authors (see e.g. 
$[24,26,28,40])$, erroneous results can be produced by truncating the set (11) to the $p$ th oscillator only, i.e. by letting $X_{i}=$ $0, \forall i \neq p$, when studying the $p$ th mode. NNMs offers a clean framework to derive a single oscillator equation which captures the correct trend of non-linearity [40]. This is recalled in the next section, where the analytical expression of the coefficient which dictates the trend of non-linearity is given.

\section{Trend of non-linearity}

\subsection{NNMs and real normal form theory}

NNMs, which are invariant manifolds in phase space [39], have been defined with the objective of embedding the main dynamical features of a $N$-dof system into a single non-linear equation, hence providing accurate ROMs for non-linear analysis/synthesis. It has been shown that, thanks to real normal form theory, NNMs also provide a clean framework to properly truncate non-linear PDEs [40]. The main results obtained in [40] are here briefly recalled before applying them to the shallow shell dynamical equations.

Normal form theory relies on the fundamental theorems of Poincaré and Poincaré-Dulac [43-45]. The main idea of Poincaré was to identify and cancel all the non-linear terms, in a dynamical systems, that do not have a prime importance on the dynamics itself. To attain this objective, successive non-linear change of coordinates are analytically computed. The same method has been applied to vibratory systems in [40], where a real formulation was kept throughout the calculations, instead of the usual complex form used by the mathematicians, and, in the mechanical context, by Jézéquel and Lamarque [46]. Real normal form here means that, at linear stage, oscillator-block of the form:

$\left(\begin{array}{c}\dot{X}_{p} \\ \dot{Y}_{p}\end{array}\right)=\left(\begin{array}{cc}0 & 1 \\ -\omega_{p}^{2} & 0\end{array}\right)\left(\begin{array}{c}X_{p} \\ Y_{p}\end{array}\right)$,

where $X_{p}$ is the modal displacement and $Y_{p}=\dot{X}_{p}$ the associated modal velocity, are kept instead of the usual complex formulation with a diagonal matrix with the two complex conjugate eigenvalues $\pm \mathrm{i} \omega_{p}$. The main advantage of doing so is that it allows one to keep the real oscillator form throughout the calculations: dynamical equations will always begin with $\ddot{X}+\omega^{2} X+\cdots$, which is more readable for the mechanicians community.

By application of Poincaré and Poincaré-Dulac's theorem, a non-linear change of coordinates is computed, allowing one to pass from the modal $\left(X_{p}, Y_{p}\right)$ coordinates to new-defined normal $\left(R_{p}, S_{p}\right)$ coordinates (where $R_{p}$ is homogeneous to a displacement and $S_{p}$ to a velocity), linked to invariant manifold. It formally reads:

$\left(\begin{array}{c}X_{p} \\ Y_{p}\end{array}\right)=\left(\begin{array}{c}R_{p} \\ S_{p}\end{array}\right)+\left(\begin{array}{c}P_{p}^{(3)}\left(R_{i}, S_{i}\right) \\ Q_{p}^{(3)}\left(R_{i}, S_{i}\right)\end{array}\right)$,

where $P_{p}^{(3)}$ and $Q_{p}^{(3)}$ are polynomials of the third order, whose complete expression is given in [40], which is not recalled here for the sake of brevity. Substituting for (15) in the original dynamical equations (11) leads to express the dynamics into an invariant-based span of the phase space. After this operation, proper truncations can be realized, as non-resonant coupling terms between oscillators have been cancelled. Keeping a single non-linear mode (by keeping the couple $\left(R_{p}, S_{p}\right)$ as master coordinates and by letting: $\left.\forall k \neq p: R_{k}=S_{k}=0\right)$ predicts the correct trend of non-linearity, as it has been demonstrated and numerically verified on continuous beam-like systems [40,47]. Comparisons have also been drawn in [40] with other asymptotic methods, such as the center manifold-based computation of NNMs proposed by Shaw and Pierre or the direct method (multiple scales directly into the PDE of motion) proposed by Nayfeh and Lacarbonara [24].

This formalism is here applied, in the next subsection, to derive the analytical expression of the coefficient dictating the trend of non-linearity for thin shallow shells. It is worth mentioning that Pellicano et al. also use normal form theory in [29], probably under its usual complex formulation, to predict the trend of non-linearity as function of the geometry for circular cylindrical shells. However, they did not mention the link to the NNM formulation, although it is formally the same $[40,46]$.

\subsection{Hardening/softening behaviour}

The dynamics onto the $p$ th NNM is governed, in terms of the new normal coordinate $R_{p}$, by the following equation [40]:

$\ddot{R}_{p}+\omega_{p}^{2} R_{p}+\left(A_{p p p}^{p}+\varepsilon_{\mathrm{c}} \Gamma_{p p p}^{p}\right) R_{p}^{3}+B_{p p p}^{p} R_{p} \dot{R}_{p}^{2}=0$,

where $A_{p p p}^{p}$ and $B_{p p p}^{p}$ depends on the quadratic coefficients. Explicit expressions of $A_{p p p}^{p}$ and $B_{p p p}^{p}$ are given in [40] in the most general case of an assembly of $N$ oscillators with nonlinear quadratic and cubic terms. Here is only provided, for brevity, their complete analytical expressions with respect to the coefficients $\left\{\beta_{i j}^{p}, \Gamma_{i j k}^{p}\right\}$ computed in the specific case of a shallow spherical shell, in Eqs. (19)-(20). A first-order perturbative development allows definition of the angular frequency of free oscillations $\omega_{\mathrm{NL}}$, connected to the natural frequency $\omega_{p}$ by the relation:

$\omega_{\mathrm{NL}}=\omega_{p}\left(1+T_{p} a^{2}\right)$

where $a$ is the amplitude of the response of the $p$ th NNM and $T_{p}$ the coefficient governing the trend of non-linearity. If $T_{p}>0$, then hardening behaviour occurs, whereas $T_{p}<0$ implies softening behaviour. The analytical expression for $T_{p}$ is now obtained by application of any perturbative method to Eq. (16). It gives:

$T_{p}=\frac{1}{8 \omega_{p}^{2}}\left[3\left(A_{p p p}^{p}+\varepsilon_{\mathrm{c}} \Gamma_{p p p}^{p}\right)+\omega_{p}^{2} B_{p p p}^{p}\right]$,

where

$$
\begin{aligned}
& A_{p p p}^{p}=\varepsilon_{\mathrm{q}}^{2} \sum_{l=1}^{+\infty} \frac{2 \omega_{p}^{2}-\omega_{l}^{2}}{D_{p l}}\left(\beta_{p l}^{p}+\beta_{l p}^{p}\right) \beta_{p p}^{l}, \\
& B_{p p p}^{p}=\varepsilon_{\mathrm{q}}^{2} \sum_{l=1}^{+\infty} \frac{2}{D_{p l}}\left(\beta_{p l}^{p}+\beta_{l p}^{p}\right) \beta_{p p}^{l},
\end{aligned}
$$


and

$D_{p l}=\omega_{l}^{2}\left(\omega_{l}-2 \omega_{p}\right)\left(\omega_{l}+2 \omega_{p}\right)$.

One can see the influence of the quadratic coefficients through the $A_{p p p}^{p}$ and $B_{p p p}^{p}$ terms. They are able to substantially modify the trend of non-linearity predicted by keeping a single linear mode. The remainder of the study will consist of numerical computations of $T_{p}$, as function of the aspect ratio $\kappa$, which is the only free parameter related to geometry.

A numerical study of the effect of the geometry on the nonlinear coefficients $\left\{\beta_{i j}^{p}\right\}$ and $\left\{\Gamma_{i j k}^{p}\right\}$ is provided in [41]. It shows that these coefficients display a very slight dependence on curvature. This is the consequence of the slight dependence of the mode shapes with the aspect ratio, as the non-linear coefficients are computed from integrals involving the mode shape functions (Eqs. (12)-(13)). Hence, the main effect of the shell's geometry on the trend of non-linearity is described by the relative variations of the eigenfrequencies, shown on Fig. 2. This will be discussed in detail in the next sections where the results are presented for different modes. The next two subsections are concerned with comments on the analytical formula with respect to modal truncation and internal resonance.

\subsection{Modal truncation}

Using a single NNM to predict the trend of non-linearity gives insight on the choice of the linear modes that have to be retained to construct the NNM. As shown in Eqs. (19)-(20), all the linear modes are embedded into a single non-linear mode through the infinite summations resulting in the $A_{p p p}^{p}$ and $B_{p p p}^{p}$ terms. In practice, these summations have to be truncated to a finite number $N$ of modes. The main advantage of the analytical formula is that one is able to choose for truncation the modes which have a real influence on the hardening/softening behaviour. As it appears in Eqs. (19)-(20), when studying the trend of non-linearity of the $p$ th mode, one has to keep all the $l$ modes such that $\beta_{p p}^{l} \neq 0$, and $\beta_{p l}^{p} \neq 0$ or $\beta_{l p}^{p} \neq 0$.

As shown in Ref. [41], a number of coefficients $\left\{\beta_{i j}^{p}\right\}_{p, i, j} \geqslant 1$ are equal to zero due to the rotational symmetry of the structure. The conditions for these quadratic coefficients to be non-zero are expressed in terms of the number of nodal diameters $k_{l}$ and $k_{p}$ of the $l$ and $p$ modes. They read:

(i) $\beta_{p p}^{l} \neq 0$ if $k_{l} \in\left\{2 k_{p}, 0\right\}$,

(ii) $\beta_{p l}^{p} \neq 0$ or $\beta_{l p}^{p} \neq 0$ if $k_{p} \in\left\{k_{l}+k_{p},\left|k_{l}-k_{p}\right|\right\}$.

These rules show that two classes of modes have to be retained when studying the trend of non-linearity of the $p$ th mode: axisymmetric $\left(k_{l}=0\right)$ as well as asymmetric modes having twice the number of nodal diameters $\left(k_{l}=2 k_{p}\right)$. No other mode has an influence on the trend of non-linearity.

In the remainder of the study, $N$ will refer to the number of modes retained in this specific subset composed of the pertinent ones with respect to the trend of non-linearity. When $N=1$, formula (18) reduces to that obtained when keeping a single-mode in the truncation, thus recovering earlier results presented with this assumption [13-16,21]. Modal truncation will be studied by increasing $N$ until convergence. Thanks to the analytical formula, one is awaiting better convergence results than if a blind modal truncation had been used, since modes having no influence on the trend of non-linearity are identified and already discarded.

The main time-consuming task in the numerical effort, when $N$ becomes large, is the computation of all the quadratic coefficients $\left\{\beta_{i j}^{k}\right\}_{k, i, j=1 \ldots N}$, needed to construct the summations. In order to save time, advantage has been taken of their slight dependence with respect to the aspect ratio $\kappa$ : quadratic coefficients are kept constant on small $\kappa$-intervals, instead of computing them for each value of the aspect ratio. This approximation has been verified and is assessed by the continuity of $T_{p}$ at the edges of the intervals where the $\left\{\beta_{i j}^{k}\right\}$ are computed. If the discontinuity is too important, then the interval is taken smaller.

\subsection{Internal resonance}

An internal resonance leads to a divergence in asymptotic developments because of small denominators. The calculation of $T_{p}$ presented before assumes no internal resonance between $\omega_{p}$ and another eigenfrequency $\omega_{l}$ of the system. Now internal resonances are likely to appear in the eigenspectrum when varying the aspect ratio $\kappa$. If all the calculations needed to compute $T_{p}$ are not possible when an internal resonance relationship is perfectly fulfilled, it is still possible in the vicinity of it, and thus will be realized.

Another interesting point arising from the analytical formula of $T_{p}$, Eq. (21), is that there is only one kind of internal resonance, namely $2: 1$ resonance, which have an influence on the trend of non-linearity. When studying the $p$ th mode, only the $l$ th modes, whose eigenfrequencies are such that $\omega_{l}=2 \omega_{p}$, are able to significantly change the value of $T_{p}$. Other second-order internal resonances, e.g. $\omega_{p}=2 \omega_{l}$, or $\omega_{l}+\omega_{m}=\omega_{p}$, are not able to produce a small denominator and to change the value of $T_{p}$. Finally, third-order internal resonances have no influence since only the first-order correction to the backbone curve is studied (Eq. (17)).

In case of 2:1 internal resonance, the normal form of the problem, which govern the dynamics onto the corresponding NNMs, must retain the two $p$ and $l$ oscillator equations, as well as the resonant coupling quadratic terms $\left(\beta_{p l}^{p}\right.$ and $\left.\beta_{p p}^{l}\right)$. In this case, a first-order perturbative study shows that only coupled solutions are possible (see Appendix A, or [48]). Thus, the trend of non-linearity, which is a notion associated to the backbone curve of a single oscillator, does not have anymore meaning. In the following sections, concerned with the numerical results, it will be seen that the value of $T_{p}$ diverges to infinity when encountering a 2:1 internal resonance. On a physical viewpoint, these values does not have sense, and the calculation presented here is not valid in a small interval around the resonance value. This phenomenon can be compared to the results presented by Pellicano et al. [29], where boundaries between hardening and softening regions are due to $2: 1$ internal resonance, as well as the results presented by Arafat and Nayfeh for suspended cables [38] and Rega et al. [37] for buckled beams and cables, where 
the divergence of $T_{p}$ in the vicinity of $2: 1$ resonance is also shown.

\section{Asymmetric modes}

In this section, results for purely asymmetric modes (i.e. modes with $k$ nodal diameters and zero nodal circles), are presented. The first subsection is devoted to the fundamental $(2,0)$ mode. Then three other asymmetric modes are investigated: $(3,0),(4,0)$ and $(7,0)$. The Poisson ratio is $v=0.33$ for all the presented results. It is recalled that the peculiarity of these modes is their slight dependence on curvature (see Fig. 2).

\subsection{Fundamental mode}

The fundamental mode, with the lowest eigenfrequency, is the $(2,0)$ mode, for any value of the aspect ratio $\kappa$. The pertinent modes, able to influence the trend of non-linearity $T_{(2,0)}$, are axisymmetric modes and asymmetric modes with 4 nodal diameters. Among these, only the first $(0,1)$ axisymmetric mode, may present a 2:1 resonance with $(2,0)$ : this occurs for $\kappa=2.687$.

The results are presented on Fig. 3, left. The hardening behaviour, typical of flat plates, is found for $\kappa=0$. Then, in favour of the 2:1 resonance with $(0,1)$, the behaviour becomes softening at $\kappa=2.687$, and returns to hardening at $\kappa=4.98$. A legitimate question is to know if this softening behaviour could be experimentally observed. As stated in Section 3.4, in the vicinity of the 2:1 resonance, only coupled solutions exist and the quantity $T_{p}$ loses its meaning. A difficult problem appear with the fact that the size of the interval where only coupled solutions exist, is not provided by a perturbative solution, since no method quantify exactly how an $\varepsilon$ should be small to clearly state that the relationship: $2 \omega_{p}=\omega_{l}+\varepsilon$, is fulfilled.

Here, we can only conjecture that at $\kappa=4.98,2 \omega_{(2,0)}=10.54$, and $\omega_{(0,1)}=11.64$; which is certainly near enough to say that energy transfer will occur and thus the softening region will be difficult to observe.

The dotted line represents the solution given by the singlemode approximation $(N=1)$. As it could be expected from the behaviour of $\omega_{(2,0)}$ with $\kappa$, the single-mode approximation predicts a hardening behaviour, with a very slight decrease as $\kappa$ increases. This erroneous result is corrected as soon as the first axisymmetric mode is added in the truncation. A twomode truncation $(N=2$, with $(2,0)$ and $(0,1))$ gives excellent results, undistinguishable for small values of $\kappa$ from the solution represented, obtained with $N=12$. The 12 modes retained are: $(2,0),(4,0),(4,1),(4,2),(0,1),(0,2),(0,3)$ and $(0,4)$, with their companion modes for asymmetric configurations.

Finally, the coefficient $T_{(2,0)}$ crosses the $x$-axis at $\kappa=1871$, but stays at very small values around $10^{-3}$. Although for large values of $\kappa$, a softening behaviour is strictly speaking predicted, it is likely to observe a neutral behaviour, due to the smallness of $T_{(2,0)}$.

A two-dimensional representation is also proposed (Fig. 3, right), where the variables $x=a / R$ and $y=h / a$ have been chosen. The assumptions of shallowness allows limitation of the $x$-axis to 0.3 , as proposed in [41,49]. On the $y$-axis, $R \rightarrow+\infty$, and thus the hardening behaviour (gray-shaded region) of a plate is found. These two-dimensional maps will be sometimes used, although they are not completely representative as only one geometrical parameter is free.

\subsection{Modes $(3,0),(4,0)$ and $(7,0)$}

When increasing the number of nodal diameters $k_{p}$ of the studied mode, the number of possible 2:1 resonances with other modes increases (see Fig. 2). This constitutes the main difference with the precedent case.

For mode $(3,0)$, there is still only one possible $2: 1$ internal resonance, with $(0,1)$, which occurs at $\kappa=49.69$. This results in a change of non-linear behaviour, see Fig. 4. Contrary to the fundamental mode, the behaviour stay softening after this resonance. Finally, $T_{(3,0)}$ tends to zero as $\kappa$ tends to infinity. The result shown on Fig. 4 has been obtained with $N=13$ modes, namely: $(3,0),(6,0)-(6,2)$, and $(0,1)-(0,5)$. The singlemode truncation predicts everywhere a hardening behaviour. This is corrected as soon as the first axisymmetric mode is included in the truncation, to show that for $\kappa \geqslant 49.69$, softening behaviour occurs. Once again, a two-mode model, with $(3,0)$ and $(0,1)$ only, gives excellent results.

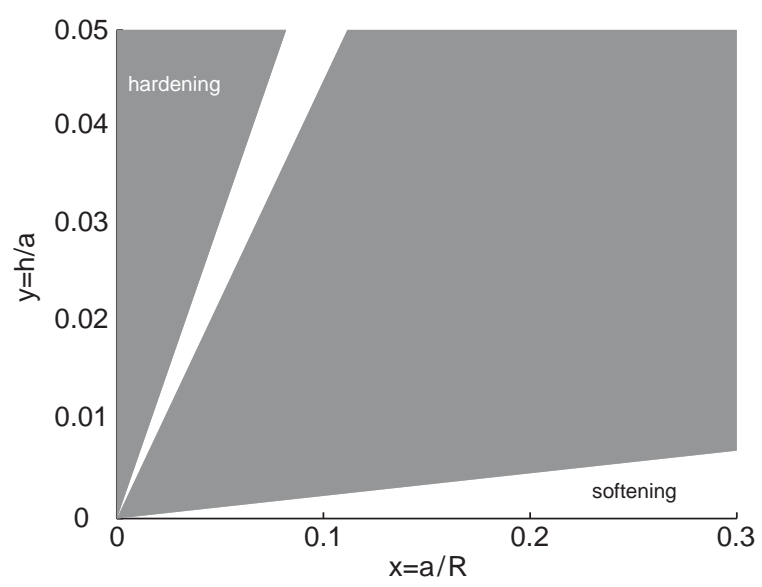

Fig. 3. Trend of non-linearity for the fundamental mode $(2,0)$. Left: Variation of $T_{(2,0)}$ with the aspect ratio $\kappa$. Right: Same result on a two-dimensional map. 


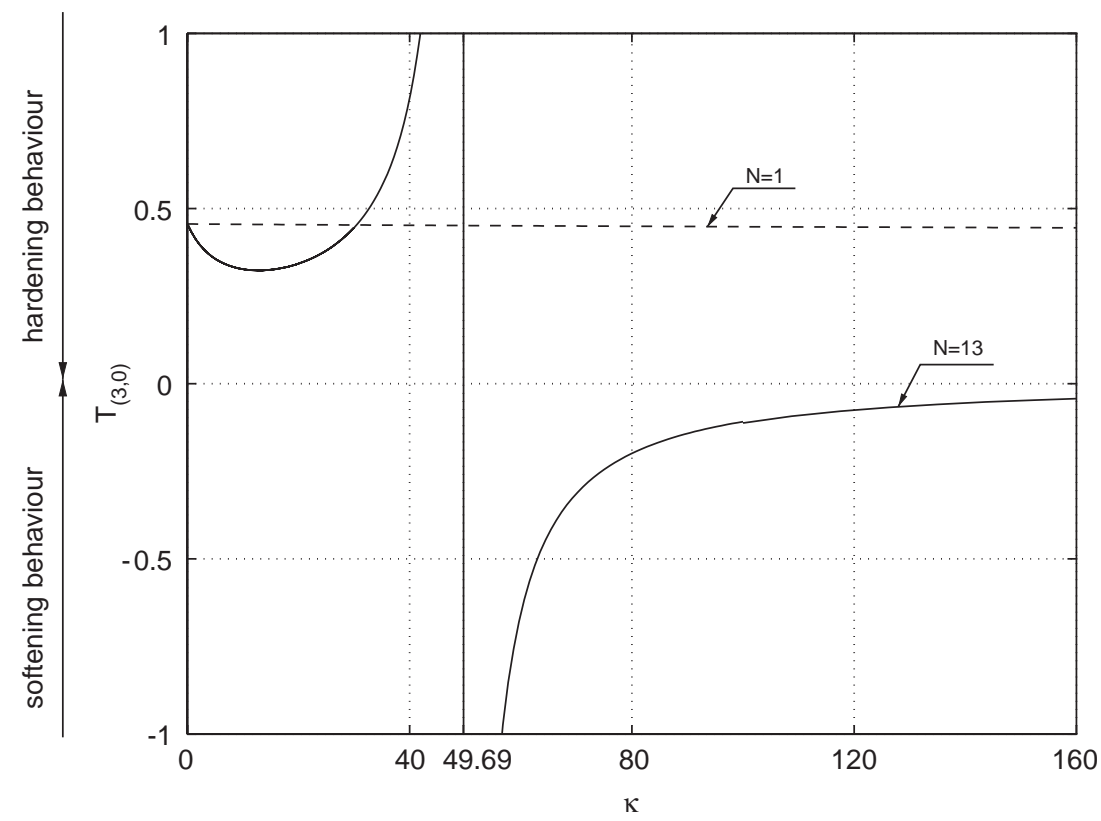

Fig. 4. Trend of non-linearity for mode $(3,0)$.

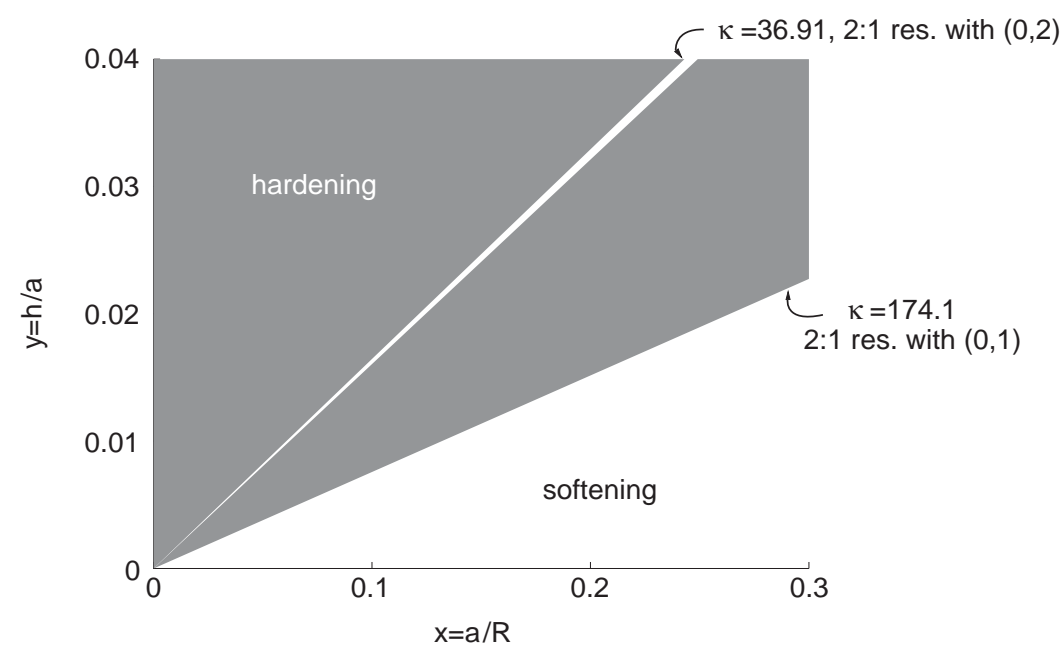

Fig. 5. Map of non-linear behaviour for mode $(4,0)$.

For mode $(4,0)$, two 2:1 internal resonances are now possible: with mode $(0,2)$ at $\kappa=36.91$, and with mode $(0,1)$ at $\kappa=174.1$. The results for the trend of non-linearity are shown on a twodimensional plot, Fig. 5. The softening region caused by the 2:1 resonance with $(0,2)$ is very narrow and must be unobservable. The non-linearity settles down in a softening behaviour after the $2: 1$ resonance with mode $(0,1)$, i.e. for $\kappa \geqslant 174.1$. Finally, the behaviour tends to be neutral when $\kappa$ tends to infinity.

The effect of the number of modes retained is shown on Fig. 6. The single-mode approximation gives once again erroneous result and predicts hardening behaviour. A two-mode truncation, including modes $(4,0)$ and $(0,1)$ is shown. The first $2: 1$ resonance is naturally missed, but the general behaviour is correctly predicted. At least a three-mode truncation must be used in this case, in order to detect the two 2:1 resonances.
Finally, mode $(7,0)$ presents three possible $2: 1$ internal resonances:

- with mode $(0,3)$ at $\kappa=868.2$,

- with mode $(0,2)$ at $\kappa=1503$,

- with mode $(0,1)$ at $\kappa=1644$.

The resulting behaviour of $T_{(7,0)}$ is shown on Fig. 7. The general behaviour is of the hardening type, except on very little parameter intervals, until the last $2: 1$ resonance, where the behaviour becomes definitively softening. The asymptotic behaviour, for very large values of $\kappa$, shows that $T_{(7,0)}$ tends to zero. The computation has been realized with $N=11$ modes, namely: $(7,0),(14,0)$ and $(14,1),(0,1)-(0,5)$.

From these results, some general rules can be deduced on the behaviour of purely asymmetric modes as a function 


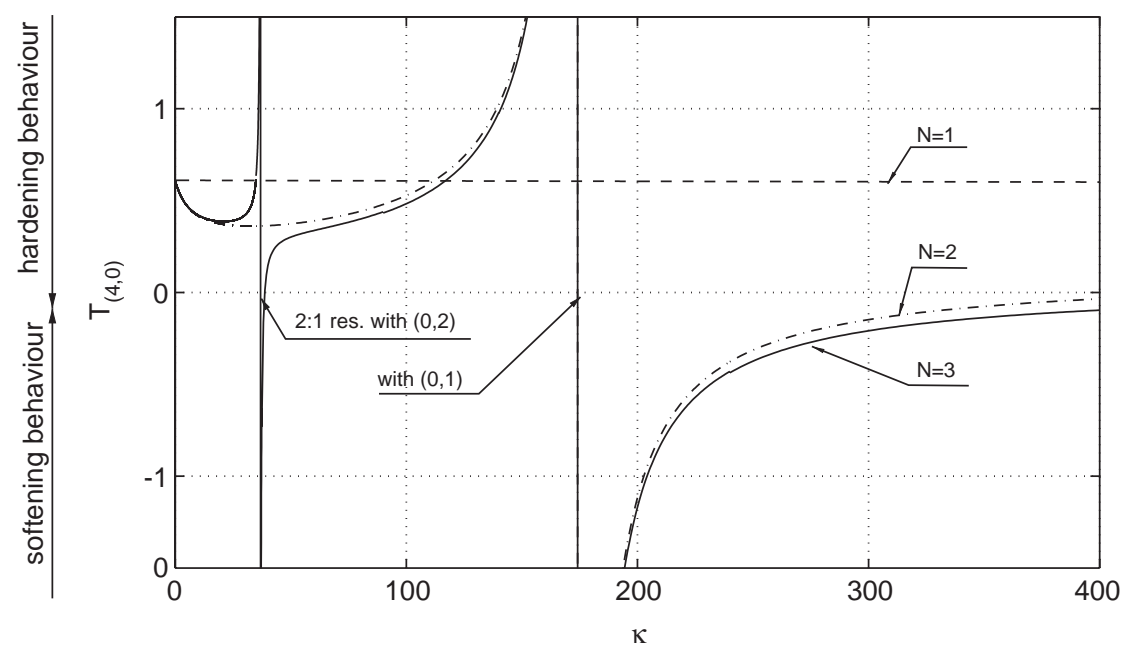

Fig. 6. Effect of the number of modes retained in the summations for the trend of non-linearity of mode $(4,0)$. Dashed line $(N=1)$ : single-mode approximation. Dash-dotted line $(N=2)$ : $(4,0)$ and $(0,1)$. Solid line $(N=3):(4,0) ;(0,1)$ and $(0,2)$.

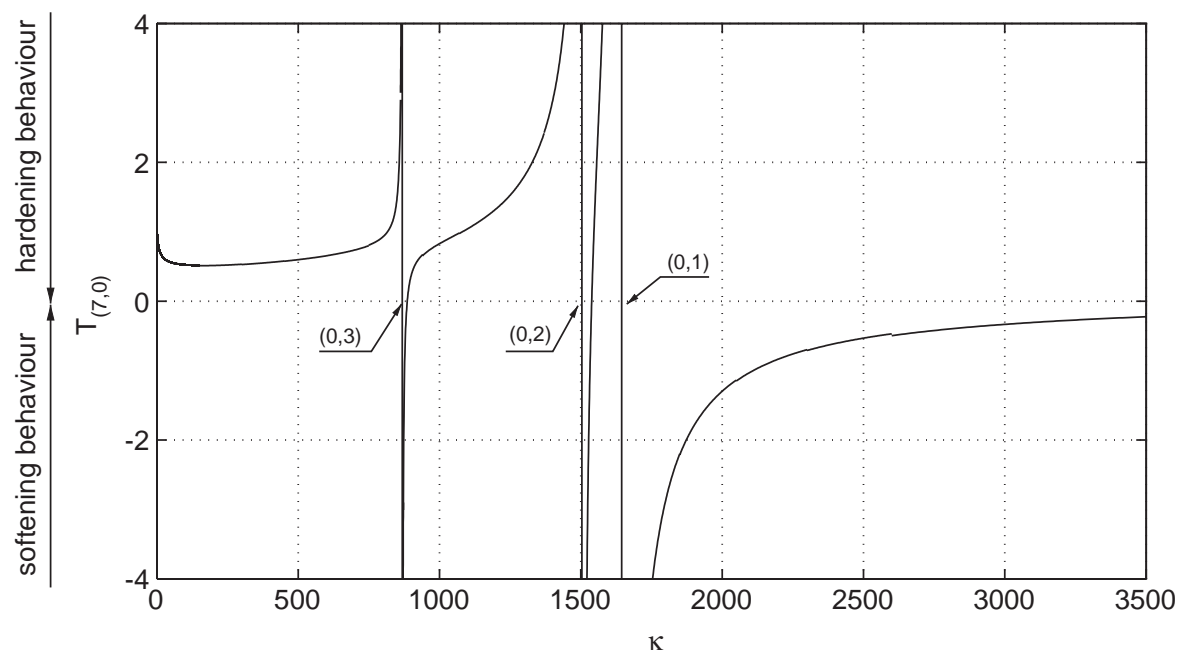

Fig. 7. Trend of non-linearity for mode (7,0). The 2:1 internal resonances with the axisymmetric modes are indicated. $N=11$ is used for the computation.

of the geometry:

- The fundamental importance of axisymmetric modes on nonlinear behaviour of asymmetric ones has been underlined.

- Mode $(2,0)$ generally display hardening behaviour, except on a narrow region after the $2: 1$ resonance with mode $(0,1)$. Then the behaviour tends to be neutral.

- For all other purely asymmetric modes, hardening behaviour is observed until the $2: 1$ resonance with mode $(0,1)$, where softening behaviour settles down.

- The trend of non-linearity tends to zero as $\kappa$ tends to infinity. The decrease of the softening behaviour with curvature has already been noticed in [16-19], for axisymmetric modes only.

- The more nodal diameters the mode has, the more $2: 1$ internal resonances are possible. However, these resonances induce softening behaviour on a narrow region which is certainly unobservable.

\section{Axisymmetric modes}

This section is devoted to presentation of the results for two axisymmetric modes: $(0,1)$ and $(0,2)$. The rules (i) and (ii) (Section 3.3 ), precizing the modes to select for computation, indicate that only axisymmetric modes have to be kept.

The main difference with previous section is the behaviour of the eigenfrequencies with respect to $\kappa$. As it can be seen on Fig. 2, axisymmetric eigenfrequencies increase with curvature. Hence, an infinity of 2:1 internal resonances are now possible, with all the other axisymmetric modes.

The result of computation is shown on Fig. 8 for mode $(0,1)$. It can be seen that the effect of the geometry-the increase of $\kappa$-is much more pronounced than for the asymmetric modes: the initial hardening behaviour $(\kappa=0)$ becomes softening at $\kappa=1.93$, and not because of a $2: 1$ internal resonance. Two resonances, leading to a change in behaviour, are then shown: at $\kappa=35.97$, where the following relationship is fulfilled: 


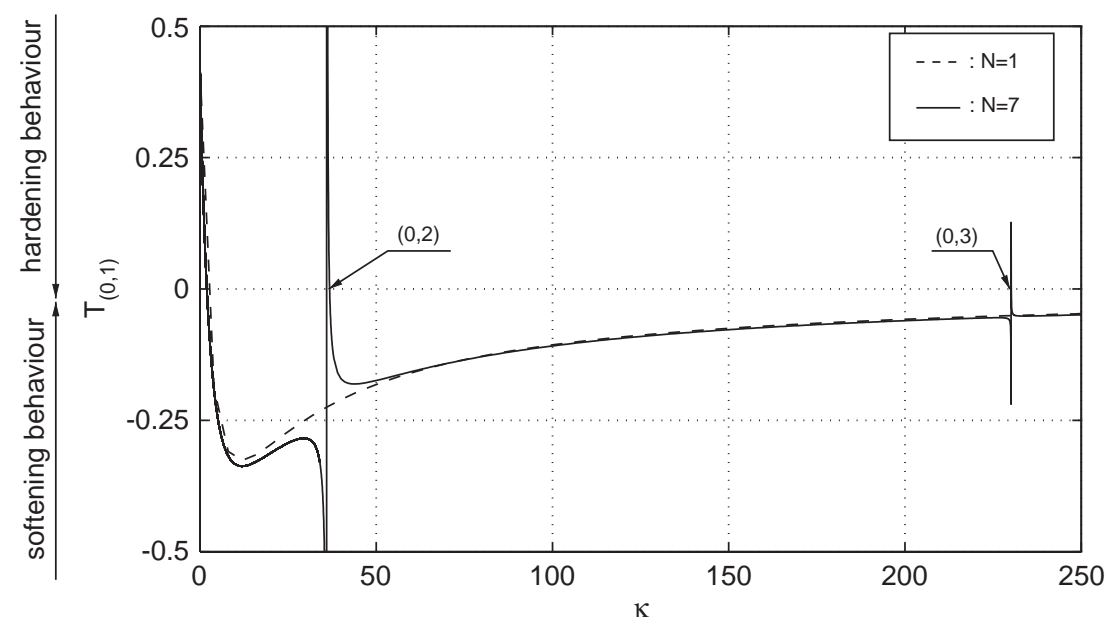

Fig. 8. Trend of non-linearity for mode $(0,1)$. Dashed line $(N=1)$ : single-mode approximation. Solid line: the seventh first axisymmetric modes are kept for computation.

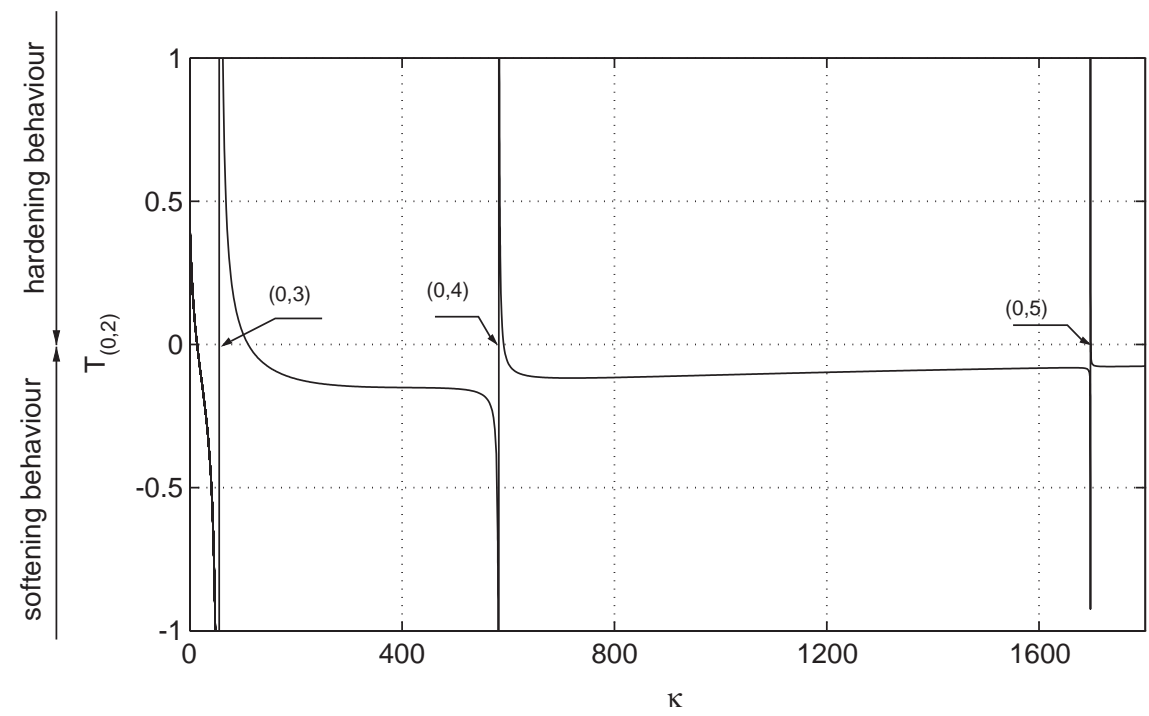

Fig. 9. Trend of non-linearity for mode $(0,2)$, computed with $N=7$ modes.

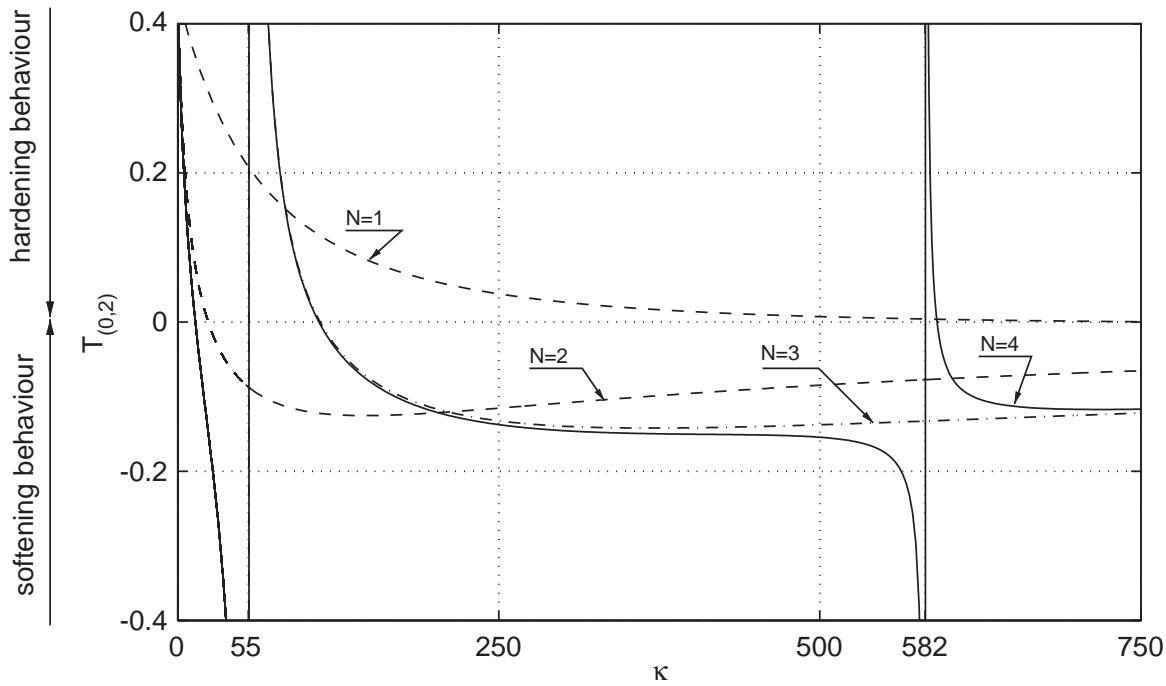

Fig. 10. Effect of the number of modes retained in the summations for the trend of non-linearity of mode $(0,2)$. Dashed lines: $N=1$ (mode $(0,2)$ only), and $N=2:(0,1)+(0,2)$. Dash-dotted line $(N=3):(0,1)$ to $(0,3)$. Solid line $(N=4):(0,1)$ to $(0,4)$. 
$2 \omega_{(0,1)}=\omega_{(0,2)}=43.21$. Then at $\kappa=230.1$, where $2: 1$ resonance occurs with mode $(0,3)$. These $2: 1$ resonances lead to a return to hardening behaviour. However, it occurs on a very little interval, which is already negligible for the resonance with $(0,2)$, and completely negligible for $(0,3)$. The next $2: 1$ resonances (with $(0,4)$ at $\kappa=756.9$, with $(0,5)$ at $\kappa=1871.5 \ldots)$ occur on intervals which are always smaller and thus are not shown.

Single-mode prediction is also shown on Fig. 8. Although the 2:1 resonances are missed, the general behaviour is correctly predicted: change from hardening to softening due to curvature is found at $\kappa=1.95$ instead of $\kappa=1.93$, and the asymptotic behaviour, which becomes neutral when $\kappa$ tends to infinity, is recovered. These results show that for the specific case of the fundamental axisymmetric mode, the single-mode approximation, used in the precedent studies $[13,14,16,17]$, predicts the essential features, in spite of a too severe truncation.

The result obtained with $N=7$ for mode $(0,2)$ is shown on Fig. 9. The effect of the geometry leads to a change from hardening to softening behaviour at $\kappa=13.73$. Then the occurrence of 2:1 internal resonances lead to slight returns to hardening behaviour on small $\kappa$ intervals. The change of behaviour due to the $2: 1$ resonance with the third axisymmetric modes leads to a hardening behaviour which occurs on a non-negligible interval: $[55.4,109.9]$. The others can be neglected and thus a softening behaviour is generally predicted. Once again, when $\kappa$ tends to infinity, $T_{(0,2)}$ tends to zero.

The effect of the number of modes retained is shown on Fig. 10. The single-mode truncation predicts a hardening behaviour for every $\kappa$, which was the results found by Yasuda and Kushida [14]. This prediction is corrected as soon as the $(0,1)$ mode is kept for computation, but 2:1 resonances are missed. The result with $N=4$ is acceptable since the essential features are predicted.

From these results, some general rules can be deduced on the behaviour of axisymmetric modes:

- The effect of geometry is much more pronounced and leads to a softening behaviour which occurs rapidly, for small values of $\kappa$.
- 2:1 internal resonances with all other axisymmetric modes are possible. However, only the internal resonance with the next axisymmetric mode leads to a significant change of behaviour. The other implies a return to the hardening behaviour which occurs on a negligible interval.

- Except for the first $(0,1)$ mode, where the single-mode approximation captures the essential features, numerous axisymmetric modes have to be kept in the computation.

- The trend of non-linearity tends to zero as $\kappa$ tends to infinity.

\section{Mixed modes}

In this section, the first three mixed modes will be studied, i.e. modes $(1,1),(2,1)$ and $(3,1)$. Mixed modes are asymmetric and have at least one nodal circle. They differ from the purely asymmetric modes by the behaviour of their eigenfrequencies with respect to $\kappa$ (see Fig. 2). Hence, as it was the case for the axisymmetric modes, they are likely to present a $2: 1$ internal resonance with all possible modes satisfying the rules (i) and (ii).

The result for mode $(1,1)$ is presented on Fig. 11. As for the axisymmetric modes, the effect of geometry is important and leads to a change of behaviour for a very small value of the aspect ratio: $\kappa=5.3$. Then $2: 1$ internal resonances occurs, with modes $(2,2),(0,3),(2,3),(0,4), \ldots$. Their number is unlimited, as for the axisymmetric case. The change of behaviour occurs on very small intervals. The first one, due to 2:1 resonance with mode $(2,2)$, is hardly negligible, and the others have no chance to be experimentally measurable. It can be thus conclude that except on a very small interval $(\kappa \in[0,5.3])$, mode $(1,1)$ behaves in a softening way.

The single-mode approximation is also shown on Fig. 11. It predicts a hardening behaviour which becomes neutral when $\kappa$ tends to infinity. The converged result is obtained for $N=17$ modes, namely: $(1,1),(2,0)-(2,4),(0,1)-(0,5)$, and shows that, contrary to the precedent cases, coefficient $T_{(1,1)}$ tends to a finite value when $\kappa$ tends to infinity. Hence, the behaviour remains softening and does not becomes neutral for large values of the aspect ratio.

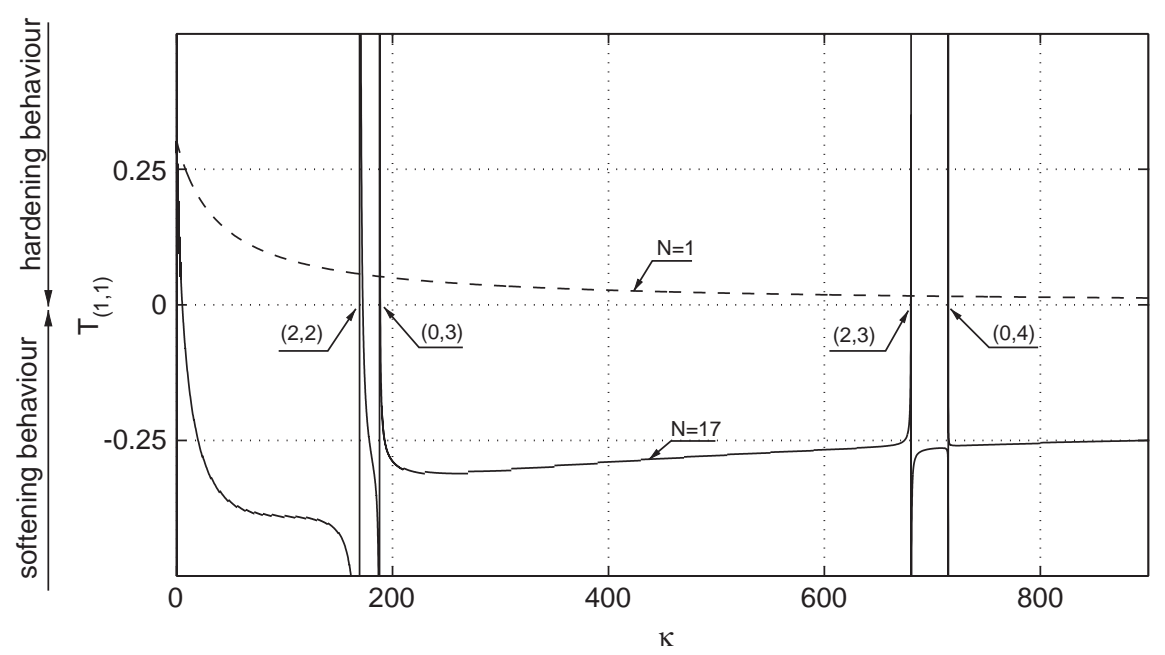

Fig. 11. Trend of non-linearity for mode (1,1). Single-mode approximation $(N=1$, dashed line $)$ and converged result $(N=17$, solid line), are represented. The occurrence of 2:1 internal resonances with modes $(2,2)(\kappa=169.4),(0,3)(\kappa=187.9),(2,3)(\kappa=680.4)$ and $(0,4)(\kappa=714.7)$ are also shown. 


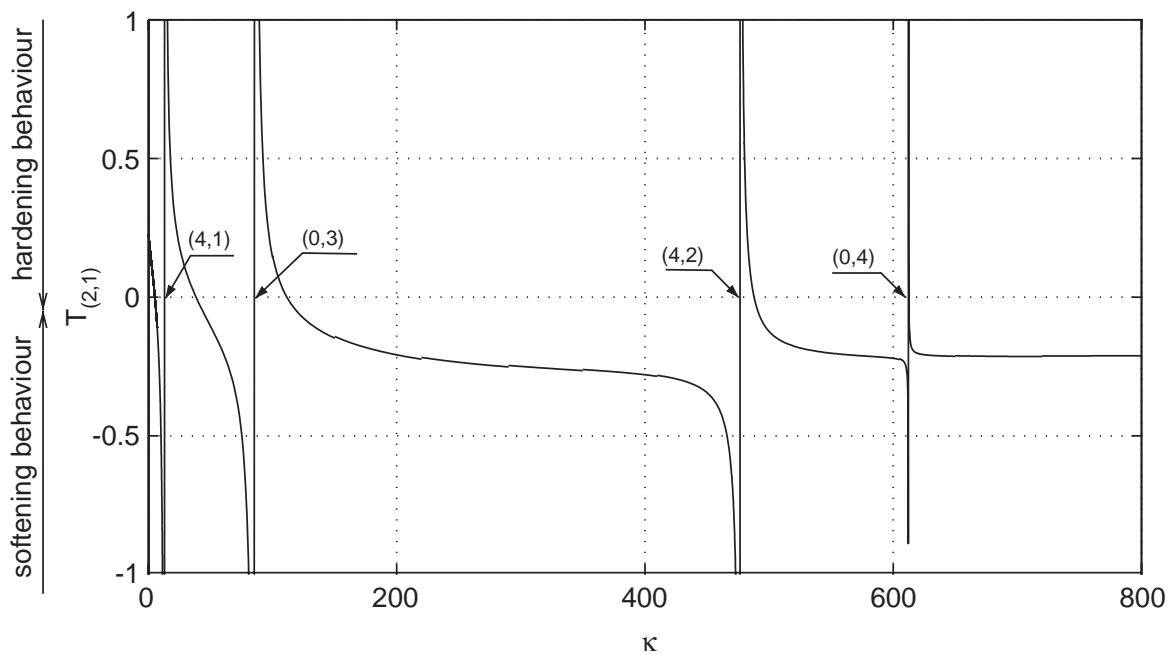

Fig. 12. Trend of non-linearity for mode $(2,1)$, obtained with $N=18$ modes: $(2,1),(4,0)-(4,4)$; and $(0,1)-(0,6)$. $2: 1$ internal resonances with modes $(4,1)$ $(\kappa=12.9),(0,3)(\kappa=85.5),(4,2)(\kappa=476.6)$ and $(0,4)(\kappa=612.2)$ are also shown.

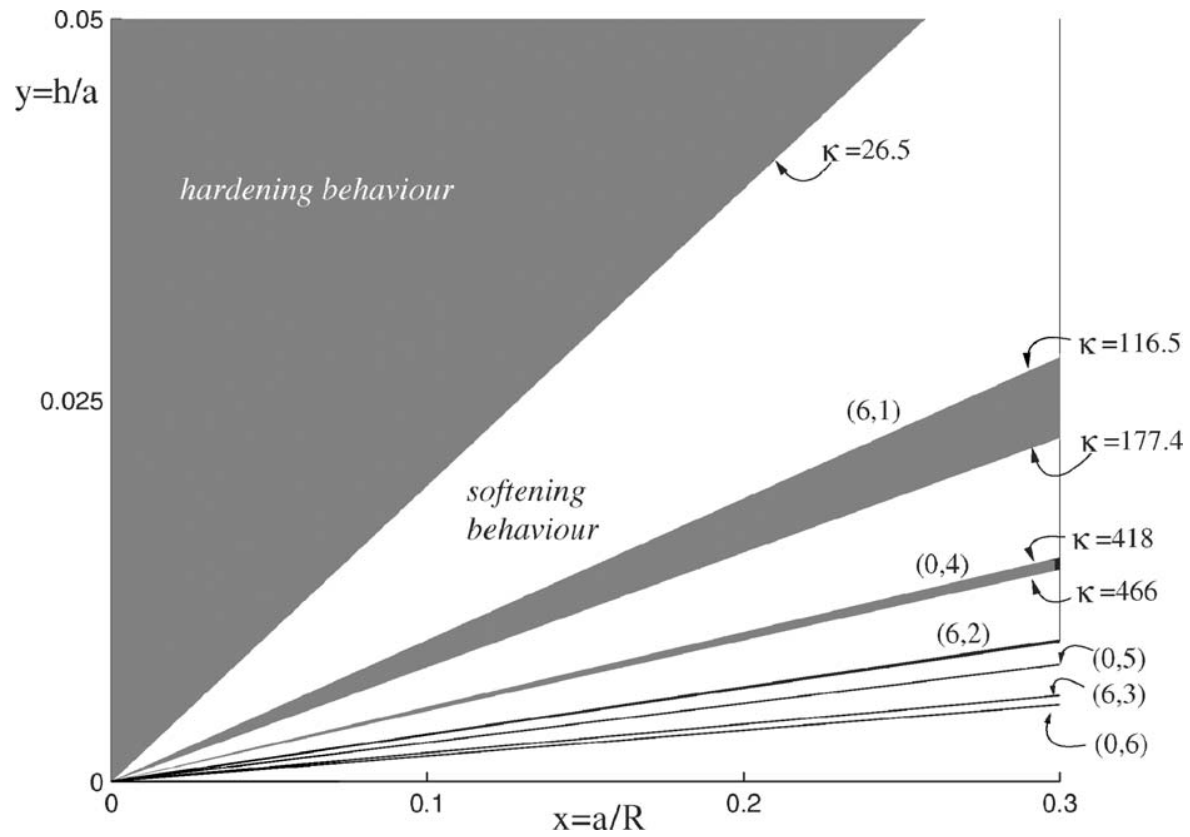

Fig. 13. Map of non-linearity for mode $(3,1) . N=16$ modes have been retained for computation: $(3,1),(6,0)-(6,3)$ and $(0,1)-(0,6)$. Other $2: 1$ resonance occurs after $(0,6)$, but are negligible and thus not represented.

The result for mode $(2,1)$ is shown on Fig. 12. The general behaviour is comparable to mode $(1,1)$ : the effect of geometry is pronounced and leads to a change from hardening to softening behaviour at $\kappa=5$. The first two 2:1 resonances implies a return to the hardening behaviour which is substantial, contrary to the precedent case. But from the resonance with mode $(0,4)$, the interval is small and the change of behaviour can be neglected. When $\kappa$ tends to infinity, $T_{(2,1)}$ tends to a finite negative value. Single-mode approximation predicts a hardening behaviour. This result was found for every mixed mode and is not reported on the figures anymore.

The result for mode $(3,1)$ is shown on a two-dimensional plot, Fig. 13. The transition from hardening to softening behaviour due to curvature occurs at $\kappa=26.5$. The first $2: 1$ internal resonance is at $\kappa=116.5$, with mode $(6,1)$. It gives a return to hardening behaviour on a non-negligible interval: $\kappa \in$ $[116.5,177.4]$. At $\kappa=177.4, \omega_{(6,1)}=129.7$ and $2 \omega_{(6,1)}=137$ : the gap between the two seems enough for allowing measurement. The next 2:1 resonance occurs with mode $(0,4)$, and gives rise to hardening behaviour for $\kappa \in[418,466]$. The other internal resonances are hardly negligible. The asymptotic behaviour shows that $T_{(3,1)}$ tends to a finite negative value for $\kappa$ to infinity.

From these results, some general rules can be deduced on the behaviour of mixed modes:

- The effect of geometry is important and leads to a softening behaviour which occurs rapidly, for small values of $\kappa$. 
- 2:1 internal resonances are numerous, but only the first ones, occurring for small $\kappa$ values are important. The others give rise to a change of behaviour on a negligible interval.

- The trend of non-linearity tends to a finite value as $\kappa$ tends to infinity.

\section{Conclusion}

Prediction of the correct trend of non-linearity for continuous structures with a curvature has been a controversial subject, mainly because erroneous results were derived on the basis of too severe truncations. The literature on circular cylindrical shell is typical of the difficulties encountered, which seems now to be definitively clarified thanks to intensive numerical computations or efficient ROMs. For shallow spherical shells, sparse results for axisymmetric modes only were available.

In this study, the trend of non-linearity for free-edge shallow spherical shells has been studied thanks to NNMs, expressed through real normal form theory. The method shows its efficiency especially in the derivation of the analytical formula of coefficient $T_{p}$, dictating the trend of non-linearity for the $p$ th mode, hence shedding light on the relevant modes to be retained for truncation, as well as on the importance of 2:1 internal resonances.

The results have been categorized into three families, as a function of the behaviour of the eigenfrequency with respect to the aspect ratio $\kappa$ of the shell. For asymmetric modes, the crucial importance of axisymmetric modes in the study of asymmetric vibrations, has been underlined. This feature is common to circular cylindrical shell, see e.g. [28]. For purely asymmetric modes, it has been shown that the behaviour was generally of the hardening type until the last 2:1 resonance, which necessarily occurs with mode $(0,1)$. After this resonance, the behaviour settles down to the softening type, except for the fundamental $(2,0)$ mode.

For axisymmetric modes, the importance of keeping numerous other axisymmetric modes in the truncation has been underlined, as compared to the precedent available studies on the subject. The huge dependence of their eigenfrequencies on curvature leads to a change from hardening to softening behaviour occurring for very small values of $\kappa$. This feature has also been found for mixed modes. These latter show the specificity of reaching a finite negative value for $T_{p}$ as $\kappa$ tends to infinity (softening behaviour), contrary to the two others families, which display a neutral behaviour for large values of the aspect ratio.

The efficiency of the method opens the way to similar studies aimed at definitively clarifying the trend of non-linearity of continuous structure with quadratic and cubic non-linearities. The formalism could easily be used to handle different boundary conditions for shallow spherical shells, as well as other curved structures such as arches.

\section{Acknowledgement}

The authors want to thank Marco Amabili for a fruitful discussion on the smoothness of the boundaries in the non-linearity map for circular cylindrical shells.

\section{Appendix A. Coupled solutions in case of 2:1 internal reso- nance}

The case of a 2:1 internal resonance between modes $p$ and $l$ is considered, i.e. the eigenfrequencies fulfil the relation: $\omega_{l} \simeq$ $2 \omega_{p}$. A detuning parameter $\sigma$ is introduced by: $\omega_{l}=2 \omega_{p}+\varepsilon \sigma$. Only the first-order perturbative solution will be expressed here, and thus cubic coefficients are omitted. The normal form of the system, which dictates the dynamics of the two resonant NNMS, writes, up to order two:

$\ddot{R}_{p}+\omega_{p}^{2} R_{p}=\varepsilon \beta_{p l}^{p} R_{l} R_{p}$

$\ddot{R}_{l}+\omega_{l}^{2} R_{l}=\varepsilon \beta_{p p}^{l} R_{p}^{2}$,

where $\varepsilon$ is used as a book-keeping device. The method of multiple scales can be used for producing the first-order solution: $R_{p}$ and $R_{l}$ are expanded as: $R_{k}=R_{k 0}+\varepsilon R_{k 1}$, with $k=l, p$. Then $R_{k 0}$ is written in polar form: $R_{k 0}=a_{k} \exp ^{\mathrm{i} \theta_{k}} \exp ^{\mathrm{i} \omega_{k} T_{0}}+$ c.c., where c.c. refers to the complex conjugate. The dynamical system governing, at the slow time scale $T_{1}$, the evolution of amplitudes $\left(a_{p}, a_{l}\right)$ and phases $\left(\theta_{p}, \theta_{l}\right)$ writes:

$$
\begin{aligned}
& a_{p}^{\prime}=\frac{\beta_{p l}^{p}}{2 \omega_{p}} a_{p} a_{l} \sin \left(\sigma T_{1}-2 \theta_{p}+\theta_{l}\right), \\
& \theta_{p}^{\prime}=-\frac{\beta_{p l}^{p}}{2 \omega_{p}} a_{l} \cos \left(\sigma T_{1}-2 \theta_{p}+\theta_{l}\right), \\
& a_{l}^{\prime}=-\frac{\beta_{p p}^{l}}{2 \omega_{l}} a_{p}^{2} \sin \left(\sigma T_{1}-2 \theta_{p}+\theta_{l}\right), \\
& \theta_{l}^{\prime}=-\frac{\beta_{p p}^{l}}{2 \omega_{l}} \frac{a_{p}^{2}}{a_{l}} \cos \left(\sigma T_{1}-2 \theta_{p}+\theta_{l}\right),
\end{aligned}
$$

where ()$^{\prime}$ refers to differentiation with respect to the slow time scale $T_{1}$. These equations are transformed into an autonomous system by defining

$\gamma=\sigma T_{1}-2 \theta_{p}+\theta_{l}$.

Finally, the fixed points are solutions of:

$$
\begin{aligned}
& \frac{\beta_{p l}^{p}}{2 \omega_{p}} a_{p} a_{l} \sin \gamma=0, \\
& \frac{\beta_{p p}^{l}}{2 \omega_{l}} a_{p}^{2} \sin \gamma=0, \\
& \sigma+\left(\frac{\beta_{p l}^{p}}{\omega_{p}} a_{l}-\frac{\beta_{p p}^{l}}{2 \omega_{l}} \frac{a_{p}^{2}}{a_{l}}\right) \cos \gamma=0 .
\end{aligned}
$$

If $\sin \gamma \neq 0$, then $a_{p}=a_{l}=0$. Thus, $\sin \gamma=0$, and $\cos \gamma= \pm 1$. The amplitudes of the solutions are then governed by the following relationship:

$$
\sigma a_{l} \pm \frac{\beta_{p l}^{p}}{\omega_{p}} a_{l}^{2} \mp \frac{\beta_{p p}^{l}}{2 \omega_{l}} a_{p}^{2}=0 .
$$


As the $p$ th mode is investigated, the question is to know if sdof solutions, such that $a_{l}=0$ and $a_{p} \neq 0$ are possible. Now letting $a_{l}=0$ in Eq. (A.5) shows that, necessarily, $a_{p}=0$. Thus, single-mode solutions do not exist anymore. Although the definition of the trend of non-linearity needs keeping the cubic-order terms, the information of the loss of existence of the sdof solution is enough to ensure that the notion of the trend of non-linearity (intrinsically connected to a sdof solution) does not have anymore meaning.

\section{References}

[1] N.H. Fletcher, Non-linear frequency shifts in quasispherical-cap shells: pitch glide in Chinese gongs, J. Acoust. Soc. Am. 78 (6) (1985) 2069-2071.

[2] C. Touzé, A. Chaigne, Lyapunov exponents from experimental time series: application to cymbal vibrations, Acta Acustica 86 (3) (2000) 557-567.

[3] A. Chaigne, C. Touzé, O. Thomas, Nonlinear vibrations and chaos in gongs and cymbals, Acoust. Sci. Technol. (Acoust. Soc. Japan) 26 (5) (2005) 403-409.

[4] S.A. Tobias, Free undamped non-linear vibrations of imperfect circular disks, Proc. Inst. Mech. Eng. 171 (1957) 691-700.

[5] N. Yamaki, Influence of large amplitudes on flexural vibrations of elastic plates, Zur Angew. Math. Mech. 41 (12) (1961) 501-510.

[6] K.A.V. Pandalai, M. Sathyamoorthy, On the modal equations of large amplitude flexural vibration of beams, plates, rings and shells, Int. J. Non-linear Mech. 3 (1973) 213-218.

[7] S. Sridhar, D.T. Mook, A.H. Nayfeh, Non-linear resonances in the forced responses of plates, Part I: symmetric responses of circular plates, J. Sound Vib. 41 (3) (1975) 359-373.

[8] C. Touzé, O. Thomas, A. Chaigne, Asymmetric non-linear forced vibrations of free-edge circular plates, Part I: theory, J. Sound Vib. 258 (4) (2002) 649-676.

[9] O. Thomas, C. Touzé, A. Chaigne, Asymmetric non-linear forced vibrations of free-edge circular plates, Part II: experiments, J. Sound Vib. 265 (5) (2003) 1075-1101.

[10] G. Prathap, K.A.V. Pandalai, The role of median surface curvature in large amplitude flexural vibrations of thin shells, J. Sound Vib. 60 (1) (1978) 119-131.

[11] O. Thomas, C. Touzé, A. Chaigne, Non-linear behavior of gongs through the dynamic of simple rods systems, in: Proceedings of the International Symposium on Musical Acoustics, Perugia, Italy, September 2001, pp. 173-178.

[12] H.A. Evensen, R.M. Evan-Iwanowsky, Dynamic response and stability of shallow spherical shells subject to time-dependant loading, AIAA J. 5 (5) (1967) 969-976.

[13] P.L. Grossman, B. Koplik, Y.-Y. Yu, Nonlinear vibrations of shallow spherical shells, ASME J. Appl. Mech. 39E (1969) 451-458.

[14] K. Yasuda, G. Kushida, Nonlinear forced oscillations of a shallow spherical shell, Bull. JSME 27 (232) (1984) 2233-2240.

[15] P.N. Singh, V. Sundarajan, Y.C. Dias, Large amplitude axisymmetric vibrations of moderately thick spherical caps, J. Sound Vib. 20 (3) (1972) 269-276.

[16] M. Sathyamoorthy, Vibrations of moderately thick shallow spherical shells at large amplitude, J. Sound Vib. 172 (1) (1994) 63-70.

[17] T.K. Varadan, K.A.V. Pandalai, Non-linear flexural oscillations of orthotropic shallow spherical shells, Comput. Struct. 9 (1978) 417-425.

[18] D. Li, A time-mode approach to non-linear vibrations of orthotropic thin shallow spherical shells, Int. J. Solids Struct. 30 (22) (1993) 3113-3128.

[19] P.B. Gonçalves, Axisymmetric vibrations of imperfect shallow spherical caps under pressure loading, J. Sound Vib. 174 (2) (1994) 249-260.

[20] D. Hui, Large-amplitude vibrations of geometrically imperfect shallow spherical shells with structural damping, AIAA J. 21 (12) (1983) 1736-1741.
[21] A.W. Leissa, A.S. Kadi, Curvature effects on shallow shell vibrations, J. Sound Vib. 16 (2) (1971) 173-187.

[22] D.K. Shin, Large amplitude free vibration behavior of doubly curved shallow open shells with simply supported edges, Comput. Struct. 62 (1) (1997) 35-49.

[23] K.A. Alhazza, Nonlinear vibrations of doubly curved cross-ply shallow shells, Ph.D. Thesis, Virginia Polytechnic Institute and State University, 2002.

[24] A.H. Nayfeh, W. Lacarbonara, On the discretization of distributedparameter systems with quadratic and cubic non-linearities, Nonlinear Dyn. 13 (1997) 203-220.

[25] M. Amabili, Non-linear vibrations of doubly curved shallow shells, Int. J. Non-linear Mech. 40 (2005) 683-710.

[26] A.H. Nayfeh, J.F. Nayfeh, D.T. Mook, On methods for continuous systems with quadratic and cubic nonlinearities, Nonlinear Dyn. 3 (1992) $145-162$.

[27] M. Amabili, F. Pellicano, M.P. Païdoussis, Non-linear vibrations of simply supported, circular cylindrical shells, coupled to quiescent fluid, J. Fluids Struct. 12 (1998) 883-918

[28] M. Amabili, F. Pellicano, M.P. Païdoussis, Non-linear dynamics and stability of circular cylindrical shells containing flowing fluid, Part II: large-amplitude vibrations without flow, J. Sound Vib. 228 (5) (1999) $1103-1124$.

[29] F. Pellicano, M. Amabili, M.P. Païdoussis, Effect of the geometry on the non-linear vibration of circular cylindrical shells, Int. J. Non-linear Mech. 37 (2002) 1181-1198.

[30] E.H. Dowell, Comments on the nonlinear vibrations of cylindrical shells, J. Fluids Struct. 12 (8) (1998) 1087-1089.

[31] M. Amabili, F. Pellicano, M.P. Païdoussis, Further comments on nonlinear vibrations of shells, J. Fluids Struct. 13 (1) (1999) 159-160.

[32] D.A. Evensen, Nonlinear vibrations of cylindrical shells-logical rationale, J. Fluids Struct. 13 (1) (1999) 161-164.

[33] M. Amabili, M.P. Païdoussis, Review of studies on geometrically nonlinear vibrations and dynamics of circular cylindrical shells and panels, with and without fluid-structure interaction, ASME Appl. Mech. Rev. 56 (4) (2003) 349-381.

[34] A. Steindl, H. Troger, Methods for dimension reduction and their applications in nonlinear dynamics, Int. J. Solids Struct. 38 (2001) 2131-2147.

[35] A.H. Nayfeh, Reduced-order models of weakly non-linear spatially continuous systems, Nonlinear Dyn. 16 (1998) 105-125.

[36] W. Lacarbonara, A theoretical and experimental investigation of nonlinear vibrations of buckled beams, Ph.D. Thesis, Virginia Polytechnic Institute and State University, 1997.

[37] G. Rega, W. Lacarbonara, A.H. Nayfeh, Reduction methods for nonlinear vibrations of spatially continuous systems with initial curvature, Solid Mech. Appl. 77 (2000) 235-246.

[38] H.N. Arafat, A.H. Nayfeh, Non-linear responses of suspended cables to primary resonance excitation, J. Sound Vib. 266 (2003) 325-354.

[39] S.W. Shaw, C. Pierre, Normal modes for non-linear vibratory systems, J. Sound Vib. 164 (1) (1993) 85-124.

[40] C. Touzé, O. Thomas, A. Chaigne, Hardening/softening behaviour in non-linear oscillations of structural systems using non-linear normal modes, J. Sound Vib. 273 (1-2) (2004) 77-101.

[41] O. Thomas, C. Touzé, A. Chaigne, Non-linear vibrations of free-edge thin spherical shells: modal interaction rules and 1:1:2 internal resonance, Int. J. Solids Struct. 42 (11-12) (2005) 3339-3373.

[42] O. Thomas, E. Luminais, C. Touzé, Non-linear modal interactions in freeedge thin spherical shells: measurements of a 1:1:2 internal resonance, in: Proceedings of the Third MIT Conference on Computational Fluid and Solid Mechanics, Boston, 2005.

[43] H. Poincaré, Les Méthodes Nouvelles de la Mécanique Céleste, Gauthiers-Villars, Paris, 1892.

[44] V.I. Arnold, Chapitres Supplémentaires de la Théorie des Équations Différentielles Ordinaires, Mir/Librairie du Globe, Paris/Moscou, 1980. 
[45] A.D. Brjuno, Analytical form of differential equations, Trans. Moscow Math. Soc. 25 (1971) 131-288.

[46] L. Jézéquel, C.H. Lamarque, Analysis of non-linear dynamical systems by the normal form theory, J. Sound Vib. 149 (3) (1991) 429-459.

[47] C. Touzé, O. Thomas, A. Huberdeau, Asymptotic non-linear normal modes for large amplitude vibrations of continuous structures, Comput. Struct. 82 (31-32) (2004) 2671-2682.
[48] A.H. Nayfeh, D.T. Mook, Nonlinear Oscillations, Wiley, New York, 1979.

[49] A. Kalnins, Effect of bending on vibrations of spherical shells, J. Acoust. Soc. Am. 36 (1) (1964) 74-81. 